\title{
Gross misinterpretation of a conditionally solvable eigenvalue equation
}

\author{
Paolo Amore* \\ Facultad de Ciencias, CUICBAS, Universidad de Colima, \\ Bernal Díaz del Castillo 340, Colima, Colima,Mexico \\ and \\ Francisco M. Fernández ${ }^{\dagger}$ \\ INIFTA, División Química Teórica, \\ Blvd. 113 y 64 (S/N), Sucursal 4, Casilla de Correo 16, \\ 1900 La Plata, Argentina
}

\begin{abstract}
We solve an eigenvalue equation that appears in several papers about a wide range of physical problems. The Frobenius method leads to a three-term recurrence relation for the coefficients of the power series that, under suitable truncation, yields exact analytical eigenvalues and eigenfunctions for particular values of a model parameter. From these solutions some researchers have derived a variety of predictions like allowed angular frequencies, allowed field intensities and the like. We also solve the eigenvalue equation numerically by means of the variational Rayleigh-Ritz method and compare the resulting eigenvalues with those provided by the truncation condition. In this way we prove that those physical predictions are merely artifacts of the truncation condition.
\end{abstract}

*e-mail: paolo@ucol.mx

${ }^{\dagger} \mathrm{e}-\mathrm{mail}$ : fernande@quimica.unlp.edu.ar 


\section{Introduction}

In a series of papers, several authors discussed a wide variety of physical models in cylindrical coordinates that, after some suitable transformations of the main dynamical (or eigenvalue) equation, can be reduced to an eigenvalue equation in the radial variable with Coulomb (or Coulomb-like) and harmonic (or harmonic-like) interactions. Through further application of the Frobenius (power-series) method they obtained a three-term recurrence relation. They stated that in order to have finite solutions (or normalizable ones) the power series should terminate and they placed a suitable truncation condition for that purpose. As a result of this truncation those authors invariably draw the conclusion that some model parameters, like the intensity of a magnetic field or the oscillator frequency, for example, should be discrete. In other words, they appeared to suggest that the eigenvalue equation has square-integrable solutions only for some particular values (allowed values) of such parameters. Several years ago, Verçin [1] derived an exact solution to the problem of two identical charged anyons moving in a plane under the influence of a static uniform magnetic field perpendicular to that plane. He argued that there are bound states if and only if the series terminates, which occurs only for certain discrete values of the magnetic field. Later, Myrheim et al 2] discussed Verçin's results with more detail finding that there are solutions for all values of the magneticfield intensity. Furtado et al [3] discussed the influence of a disclination on the spectrum of an electron or a hole in a magnetic field in the framework of the theory of defects and three-dimensional gravity of Katanaev and Volovich [4]. Although they were aware of the results derived by Myrheim et al [2], they surprisingly concluded that the cyclotron frequency and the magnetic field should depend on the quantum numbers. From these papers sprouted many others: for example, Bakke and Moraes [5] introduced a geometric model to explain the origin of the observed shallow levels in semiconductors threaded by a dislocation density and find allowed values of the oscillator frequency or of the constant $k$ 
associated to the momentum along the z-axis. Bakke and Beilish [6] obtained the bound states for a non-relativistic spin-half neutral particle under the influence of a Coulomb-like potential induced by the Lorentz symmetry breaking effects. They claimed to present a new possible scenario of studying the Lorentz symmetry breaking effects on a non-relativistic quantum system defined by a fixed space-like vector field parallel to the radial direction interacting with a uniform magnetic field along the $z$-axis. They also discussed the influence of a Coulomb-like potential induced by Lorentz symmetry violation effects on a two-dimensional harmonic oscillator and found allowed values of the cyclotron frequency. Bakke [7] discussed a model that consists of the interaction between a moving electric quadrupole moment and a magnetic field and also added a two-dimensional harmonic-oscillator potential thus obtaining allowed values of the oscillator frequency. Bakke [8] studied the bound states of a quantummechanical model given by the interaction between the electric quadrupole of a moving particle and an electric field. In two other models the author added a harmonic potential and a linear plus harmonic potential and also found allowed oscillator frequencies. Bakke and Belich [9] studied the effects of the Lorentz symmetry violation in the non-relativistic quantum dynamics of a spin- $\frac{1}{2}$ neutral particle interacting with external fields confined to a two-dimensional quantum ring and also found oscillator frequencies that depend on the quantum numbers. Fonseca and Bakke [10] proposed a model for the interaction of a magnetic quadrupole moment with electric and magnetic fields and in a second model they added a harmonic-oscillator potential finding that the angular frequency depends on the quantum numbers. Bakke and Furtado [1] studied the influence of a Coulomb-type potential on the Klein-Gordon oscillator and found that the angular frequency should depend on the quantum numbers. Vitória et al [12] analyzed the interface between a theory that goes beyond the Standard Model and quantum mechanics. Based on a line of research that deals with the violation of the Lorentz symmetry, where it is assumed that at least one privileged direction in the spacetime, they searched for a hint of a fundamental theory that goes beyond the energy scale of the Standard Model. According to the authors, this 
fundamental theory describes a spontaneous breaking of the Lorentz symmetry by a tensor background, which can be considered to be a perturbation. Then, they analyzed the interaction of a scalar particle with a Coulomb-type potential in the presence of a background of the violation of the Lorentz symmetry produced by a tensor field. Vitória and Belich [13] investigated the association of the pointlike global monopole to the point defects in elastic solids were a harmonic oscillator is immersed. They studied the topology effects of the medium on the harmonic oscillator and obtained bound states in analytical form. They also investigated the effects of the Coulomb and linear central potentials on the harmonic oscillator in an environment with a pointlike defect and concluded that the angular frequency of the harmonic oscillator is restricted to discrete values values determined by the quantum numbers of the system and of the parameter associated to the pointlike topological defect. Vitória and Belich [13] investigated the association of the pointlike global monopole to the point defects in elastic solids were a harmonic oscillator is immersed. They studied the topology effects of the medium on the harmonic oscillator and obtained bound states in analytical form. They also investigated the effects of the Coulomb and linear central potentials on the harmonic oscillator in an environment with a pointlike defect and concluded that the angular frequency of the harmonic oscillator is restricted to discrete values determined by the quantum numbers of the system and of the parameter associated to the pointlike topological defect. Vitória and Belich [14] investigated the relativistic oscillator model for spin $-1 / 2$ fermionic fields, in a background of breaking the Lorentz symmetry governed by a constant vector field inserted in the Dirac equation by non-minimal coupling and proposed two possible scenarios of Lorentz symmetry violation which induce a Coulomb type potential. They determined the relativistic energy profile for the Dirac oscillator analytically and conjecture an interesting quantum effect: the frequency of the Dirac oscillator is determined by the quantum numbers of the system and the parameters that characterize the scenarios of Lorentz symmetry violation. Vieira and Bakke [15] discussed the Aharanov-Bohm effect for the bound states of a neutral particle with a magnetic quadrupole moment that 
interacts with axial fields. They showed that the eigenvalues depend on the geometric quantum phase, which gives rise to an analog of the Ahranov-Bohm effect. In addition, they concluded that each radial mode yields a different set of allowed values of the angular frequency of the harmonic term included in the interaction of one of the models.

In other applications the radial part of the eigenvalue equation exhibits Coulomb, linear and harmonic terms. For example, Figueiredo Medeiros and Becerra de Mello [16] analyzed the relativistic quantum motion of charged spin0 and spin- $\frac{1}{2}$ particles in the presence of a uniform magnetic field and scalar potentials in the cosmic string spacetime and concluded that there must be allowed values of the cyclotron frequency. Bakke and Belich [17] studied the arising of a Rashba-like coupling, a Zeeman-like term and a Darwin-like term induced by Lorentz symmetry breaking effects in the non-relativistic quantum dynamics of a spin- $\frac{1}{2}$ neutral particle interacting with external fields arriving at the conclusion that not all the cyclotron frequencies are allowed. Finally, Hassanabadi et al [18] studied the interaction of magnetic quadrupole moment of neutral particle systems (such as atoms or molecules) with a radial electric field for non-relativistic particles in a rotating frame that tends to a uniform effective magnetic field perpendicular to the plane of motion of the neutral particle. To this list we also add some of the papers cited above [13, 14].

The case of linear plus harmonic terms has also been of interest [13] for example, Olivera [19] et al considered the hypothesis of a privileged direction in the space-time. To this end they take into account a background of the Lorentz symmetry violation determined by a fixed spacelike vector field and analyzed quantum effects of this background on the interaction of a nonrelativistic electron with a nonuniform electric field produced by a uniform electric charge distribution. They predicted the existence of allowed angular frequencies.

The purpose of this paper is to study the radial eigenvalue equation derived in those papers and investigate to which extent the truncation of the power series by means of the tree-term recurrence relation affects the physical conclusions drawn by their authors. In section 2 we outline the main equation solved in the 
papers mentioned above. In section 3 we solve the radial eigenvalue equation by means of the Frobenius method and truncation through a three-term recurrence relation for the series coefficients in order to derive analytical solutions. By means of a reliable variational method we also obtain accurate numerical eigenvalues that are compared with the analytical ones. Finally, in section 4 we summarize the main results and draw conclusions.

\section{The time-dependent equation}

In several of the papers listed above the starting point is a time-dependent quantum-mechanical equation of the form $[5,10,16,18$

$$
i \frac{\partial \psi}{\partial t}=H \psi
$$

where the Hamiltonian operator $H=H\left(\rho, \partial_{\rho}, \partial_{\varphi}, \partial_{z}\right), \partial_{q}=\partial / \partial q$, is given in cylindrical coordinates $0<\rho<\infty, 0 \leq \varphi \leq 2 \pi,-\infty<z<\infty$. Upon choosing the particular solution

$$
\psi(t, \rho, \varphi, z)=e^{-i \mathcal{E} t} e^{i j \varphi} e^{i k z} R(\rho)
$$

where $j=l=0 \pm 1 \pm 2, \ldots$ in some cases [7, 8, 10, 11, $j=l+1 / 2$ in others [5,6, 9, 16, 17] and $-\infty<k<\infty$ the authors derive an eigenvalue equation for $R(\rho)$ :

$$
H_{j k}\left(\rho, \partial_{\rho}\right) R(\rho)=\mathcal{E} R(\rho) .
$$

In passing, we mention that in most of the papers listed above the authors claim to have used units such that $\hbar=c=1$ or the like. This non-rigorous way of choosing suitable or natural units has been criticized in a recent pedagogical paper [20.

In most of those papers the motion of the particle is unbounded in the $z$ direction and the spectrum is continuous which is plainly reflected in the dependence of $\mathcal{E}$ on $k$. More precisely, a bound state requires that

$$
\iiint|\psi(t, \rho, \varphi, z)|^{2} \rho d \rho d \varphi d z<\infty
$$


but in the general example outlined above the improper integral over $z$ is divergent. However, there is interest in the quantization of the motion in the $x-y$ plane [21,22]. On the other hand, other authors consider a motion in a plane where there are truly bound states [1,2,11]. In what follows, we focus on the motion of the particle in the $x-y$ plane.

\section{Three-term recurrence relation}

In all the papers listed above the authors arrive at an eigenvalue equation of the form

$$
\begin{aligned}
\hat{L} R & =W R \\
\hat{L} & \equiv-\frac{d^{2}}{d \xi^{2}}-\frac{1}{\xi} \frac{d}{d \xi}+\frac{\gamma^{2}}{\xi^{2}}-\frac{a}{\xi}+b \xi+\xi^{2},
\end{aligned}
$$

where $\gamma, a$ and $b$ are real numbers and, in general, $\gamma$ depends on the rotational quantum number $l$.

By means of the ansatz

$$
R(\xi)=\xi^{|\gamma|} e^{-\frac{b \xi}{2}-\frac{\xi^{2}}{2}} P(\xi), P(\xi)=\sum_{j=0}^{\infty} c_{j} \xi^{j}
$$

we obtain a three-term recurrence relation for the coefficients $c_{j}$ :

$$
\begin{aligned}
c_{j+2} & =\frac{b(2 \gamma+2 j+3)-2 a}{2(j+2)(2|\gamma|+j+2)} c_{j+1}+\frac{4(2 \gamma+2 j-W+2)-b^{2}}{4(j+2)(2|\gamma|+j+2)} c_{j}, \\
j & =-1,0,1, \ldots, c_{-1}=0, c_{0}=1 .
\end{aligned}
$$

In the papers just mentioned the authors state, in one way or another, that in order to obtain bound states one has to force the termination conditions

$$
W=W^{(n, l)}=2(\gamma+n+1)-\frac{b^{2}}{4}, c_{n+1}=0, n=1,2, \ldots
$$

Clearly, under such conditions $c_{j}=0$ for all $j>n$ and $P(\xi)$ reduces to a polynomial of degree $n$. In this way, they obtain analytical expressions for the eigenvalues $W^{(n, l)}$ and the radial eigenfunctions $R^{(n, l)}(\xi)$ [1,3,5, 11, 16, 18]. For the sake of clarity and generality, in this section we use $\gamma$ instead of $l$ as an 
effective quantum number because the form of $\gamma$ is not the same in all those papers.

For example, when $n=1$ we have

$$
\begin{aligned}
W^{(1, \gamma)} & =2(\gamma+2)-\frac{b^{2}}{4}, a_{1, \gamma}^{(1)}=\frac{2 b(\gamma+1)-\sqrt{b^{2}+8(2 \gamma+1)}}{2} \\
a_{1, \gamma}^{(2)} & =\frac{2 b(\gamma+1)+\sqrt{b^{2}+8(2 \gamma+1)}}{2}
\end{aligned}
$$

or, alternatively,

$$
\begin{aligned}
& b_{1, \gamma}^{(1)}=\frac{2\left[2 a(\gamma+1)-\sqrt{a^{2}+2(2 \gamma+3)(2 \gamma+1)^{2}}\right]}{(2 \gamma+1)(2 \gamma+3)}, \\
& b_{1, \gamma}^{(2)}=\frac{2\left[2 a(\gamma+1)+\sqrt{a^{2}+2(2 \gamma+3)(2 \gamma+1)^{2}}\right]}{(2 \gamma+1)(2 \gamma+3)} .
\end{aligned}
$$

When $n=2$ we obtain a cubic equation for either $a$ or $b$; for example,

$$
\begin{aligned}
W^{(2, \gamma)}= & 2(\gamma+3)-\frac{b^{2}}{4}, \\
& 4 a^{3}-6 a^{2} b(2 \gamma+3)+a\left(b^{2}\left(12 \gamma^{2}+36 \gamma+23\right)-16(4 \gamma+3)\right) \\
& -\frac{b(2 \gamma+1)\left(b^{2}(2 \gamma+3)(2 \gamma+5)-16(4 \gamma+7)\right)}{2}=0
\end{aligned}
$$

from which we obtain either $a_{2, \gamma}(b)$ or $b_{2, \gamma}(a)$; for example, $a_{2, \gamma}^{(1)}(b), a_{2, \gamma}^{(2)}(b)$, $a_{2, \gamma}^{(3)}(b)$. In the general case we will have $n+1$ curves of the form $a_{n, \gamma}^{(i)}(b)$, $i=1,2, \ldots, n+1$. Numerical investigation suggests that all the roots are real; for example, figure 1 shows the three curves $b_{2, \gamma}^{(i)}(a)$ for three values of $\gamma$.

It is obvious to anybody familiar with conditionally solvable quantum-mechanical models 23 26] (and references therein) that the approach just described does not produce all the eigenvalues of the operator $\hat{L}$ for a given set of values of $\gamma, a$ and $b$ but only those states with polynomial factors $P(\xi)$. It is true that the approach yields an infinite number of eigenvalues $W^{(n, \gamma)}, n=0,1, \ldots$, $l=0, \pm 1, \pm 2, \ldots$, but one should understand that $W^{(n, \gamma)}$ is an eigenvalue of a set of models given by $a_{n, \gamma}^{(i)}(b), i=1,2, \ldots, n+1$ whereas $W^{\left(n^{\prime}, \gamma^{\prime}\right)}$ is an eigenvalue of a set of other models given by the parameters $a_{n^{\prime}, \gamma^{\prime}}^{\left(i^{\prime}\right)}(b), i^{\prime}=1,2, \ldots, n^{\prime}+1$. 
On the other hand, if we solve the eigenvalue equation (5) in a proper way we obtain an infinite set of eigenvalues $W_{\nu, \gamma}(a, b), \nu=0,1,2, \ldots$ for each set of real values of $a, b$ and $\gamma$. The condition that determines these allowed values of $W$ is that the corresponding radial eigenfunctions $R(\xi)$ are square integrable

$$
\int_{0}^{\infty}|R(\xi)|^{2} \xi d \xi<\infty
$$

Notice that $\nu$ is the actual radial quantum number (that labels the eigenvalues in increasing order of magnitude) whereas $n$ is just a positive integer that labels some particular solutions with polynomial factors $P(\xi)$ (and also a set of quantum mechanical models). In other words: $n$ is a fictitious quantum number used in those earlier papers as a true one $[1,3,[5,11,16,18]$.

According to the Hellmann-Feynman theorem [22,27] the actual eigenvalues $W_{\nu, \gamma}(a, b)$ of equation (5) are decreasing functions of $a$ and increasing functions of $b$

$$
\frac{\partial W}{\partial a}=-\left\langle\frac{1}{\xi}\right\rangle, \frac{\partial W}{\partial b}=\langle\xi\rangle .
$$

Therefore, for a given value of $b$ and sufficiently large values of $a$ we expect negative values of $W$ that the truncation condition fails to predict. It is not difficult to prove, from straightforward scaling [20, that

$$
W_{\nu, \gamma} \approx-\frac{a^{2}}{(2 \nu+2|\gamma|+1)^{2}}+\mathcal{O}\left(a^{-3}\right),
$$

when $a \rightarrow \infty$. What is more, we can conjecture that the pairs $\left[a_{n, \gamma}^{(i)}(b), W^{(n, \gamma)}\right]$, $i=1,2, \ldots, n+1$ are points on the curves $W_{\nu, \gamma}(a, b), \nu=0,1, \ldots, n$, respectively, for a given value of $b$.

The eigenvalue equation (5) cannot be solved exactly in the general case. In order to obtain sufficiently accurate eigenvalues of the operator $\hat{L}$ we resort to the reliable Rayleigh-Ritz variational method that is well known to yield increasingly accurate upper bounds to all the eigenvalues of the Schrödinger equation 27] (and references therein). For simplicity, we choose the basis set of non-orthogonal functions $\left\{u_{j}(\xi)=\xi^{|\gamma|+j} e^{-\frac{\xi^{2}}{2}}, j=0,1, \ldots\right\}$ and test the accuracy of these results by means of the powerful Riccati-Padé method [28]. 
As a first example, we choose $n=2, \gamma=0$ and $b=1$ so that $W=5.75$ for the three values of the model parameter $a: a_{2,0}^{(i)}, i=1,2,3$. Table 1 shows the first six eigenvalues for each of these models. We appreciate that the eigenvalue $W=$ 5.75 coming from the truncation condition (8) is the lowest eigenvalue of the first model, the second lowest eigenvalue of the second model and the third lowest eigenvalue for the third model. The truncation condition misses all the other eigenvalues of each of those models and for this reason it cannot provide the spectrum of the physical model (given by $a, b$, and $\gamma$ ) as believed by the authors of the papers listed above. This table also shows results for $a=2$ and $b=1$ that is not a point on any curve $a(b)$ stemming from the truncation condition. As said above: there are square-integrable solutions (actual bound states) for any set of real values of $a, b$ and $\gamma$. Figure 2 shows $\xi R_{\nu, 0}(\xi)^{2}$ for $\nu=0,1,2$ and $a_{2,0}^{(i)}, i=1,2,3$. In each case, the continuous line is the radial function with a polynomial factor $P(\xi)$ and the dashed lines indicate the variational results that the truncation condition does not produce. We appreciate that the truncation condition provides only one solution for each model and this figure confirms that the radial eigenfunctions that are missed by the truncation condition are also square integrable. Figure 3 shows several eigenvalues $W_{\nu, 0}(a)$ for $b=1$. The red points are results given by the truncation condition and the continuous blue line are variational results that fill the gaps leaved by such arbitrary condition. These results are most revealing because they confirm our earlier conjecture about the meaning of the pairs $\left[a_{n, \gamma}^{(i)}(b), W^{(n, \gamma)}(b)\right], n=1,2, \ldots, i=1,2, \ldots, n+1$, for a fixed value of $b$. To be clearer: the spectrum of the operator $\hat{L}$ for $\gamma=0, b=1$ and a chosen value of $a$ is given by the intersections of a vertical line with the blue lines. Such a vertical line goes through no more than only one red point at best. The obvious conclusion is that the truncation condition (8) yields only one eigenvalue of the spectrum of a given model and, consequently, the allowed cyclotron frequencies, allowed field intensities, model parameters that depend on the quantum numbers, etc., conjectured by the authors of the papers listed above, are mere artifacts of this arbitrary condition. Such claims are nonsensical from a physical point of view. 


\section{Conclusions}

In all the papers listed above $[1,3,5,11,16,18$, the authors made two basic, conceptual errors. The first one is to believe that the only possible bound states are those with polynomial factors $P(\xi)$. We have shown above that there are square-integrable solutions for model parameters $a$ and $b$ outside the curves $a_{n, \gamma}^{(i)}(b)$ stemming from the truncation condition (8) . The second one is the statement that the spectrum of the problem is given by the truncation condition (8). It is clear that this equation only provides one energy eigenvalue for a particular set of models given by the curves just mentioned. For this reason, all the claims about allowed cyclotron frequencies, allowed field intensities, model parameters that depend on the quantum numbers, etc. are nonsensical. All these conclusions stem from an arbitrary truncation condition that only produces particular bound states with no physical meaning.

\section{Acknowledgements}

The research of P.A. was supported by Sistema Nacional de Investigadores (México).

\section{References}

[1] A. Verçin, Phys. Lett. B 260 (1991) 120-124.

[2] J. Myrheim, E. Halvorsen, and A. Verçin, Phys. Lett. B 278 (1992) 171-174.

[3] C. Furtado, B. G. C da Cunha, F. Moraes, E. R. Bezerra de Mello, and V. B. Bezzerra, Phys. Lett. A 195 (1994) 90-94.

[4] M. O. Katanaev and I. V. Volovich, Ann. Phys. 216 (1992) 1-28.

[5] K. Bakke and F. Moraes, Phys. Lett. A 376 (2012) 2838-2841.

[6] K. Bakke and H. Belich, Eur. Phys. J. Plus 127 (2012) 102. 
[7] K. Bakke, Ann. Phys. 341 (2014) 86-93.

[8] K. Bakke, Int. J. Mod. Phys. A 29 (2014) 1450117.

[9] K. Bakke and H. Belich, Eur. Phys. J. Plus 129 (2014) 147.

[10] I. C. Fonseca and K. Bakke, J. Math. Phys. 56 (2015) 062107.

[11] K. Bakke and C. Furtado, Ann. Phys. 355 (2015) 48-54.

[12] L. L. Vitória, K. Bakke, and H. Belich, Ann. Phys. 399 (2018) 117-123.

[13] L. L. Vitória and H. Belich, Phys. Scr. 94 (2019) 125301.

[14] L. L. Vitória, Eur. Phys. J. Plus 135 (2020) 247.

[15] S. L. R. Vieira and K. Bakke, Phys. Rev. A 101 (2020) 032102.

[16] E. R. Figueiredo Medeiros and E. R. Bezerra de Mello, Eur. Phys. J. C 72 (2012) 2051.

[17] K. Bakke and H. Belich, Ann. Phys. (Berlin) 526 (2013) 187-194.

[18] H. Hassanabadi, M. de Montigny, and M. Hosseinpour, Ann. Phys. 412 (2020) 168040.

[19] A. S. Olivera, K. Bakke, and H. Belich, Eur. Phys. J. Plus 135 (2020) 623.

[20] F. M. Fernández, Dimensionless equations in non-relativistic quantum mechanics, arXiv:2005.05377 [quant-ph]

[21] L. D. Landau and E. M. Lifshitz, Quantum Mechanics. Non-relativistic Theory, (Pergamon, New York, 1958).

[22] C. Cohen-Tannoudji, B. Diu, and F. Laloë, Quantum Mechanics, (John Wiley \& Sons, New York, 1977).

[23] A. DeSousa Dutra, Phys. Lett. A 131 (1988) 319-321.

[24] S. Bera, B. Chakrabarti, and T. K. Das, Phys. Lett. A 381 (2017) 13561361. 


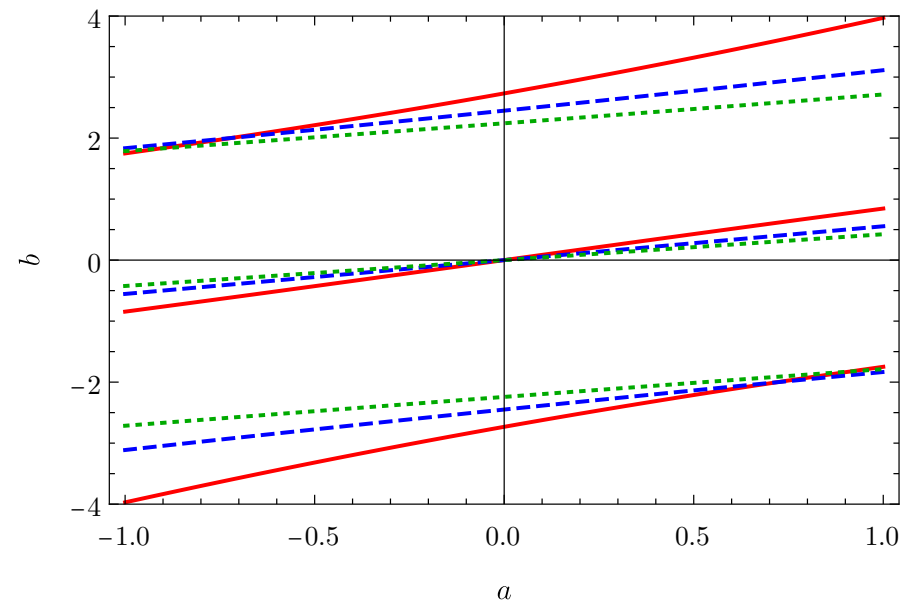

Figure 1: Curves $b_{2, \gamma}^{(i)}(a), i=1,2,3$, for $\gamma=0$ (red, continuous line), $\gamma=1 / 2$ (blue, dashed line) and $\gamma=1$ (green, dotted line)

[25] A. V. Turbiner, One-dimensional quasi-exactly solvable Schrodinger equations, arXiv:1603.02992v2

[26] P. Amore and F. M. Fernández, On some conditionally solvable quantummechanical problems, arXiv:2007.03448 [quant-ph]

[27] F. L. Pilar, Elementary Quantum Chemistry, (McGraw-Hill, New York, 1968).

[28] F. M. Fernández, Q. Ma, and R. H. Tipping, Phys. Rev. A 39 (1989) 16051609. 
Table 1: First six eigenvalues $W_{\nu, 0}(a)$ for $b=1$ and some values of $a$

\begin{tabular}{lc}
\hline$\nu$ & $W_{\nu, 0}$ \\
\hline$a_{2,0}^{(1)}=-1.940551663$ \\
\hline 0 & 5.75 \\
1 & 9.89404066 \\
2 & 14.06831985 \\
3 & 18.24977457 \\
4 & 22.4306056 \\
5 & 26.60791902 \\
\hline & $a_{2,0}^{(2)}=1.190016441$ \\
\hline 0 & -0.1664353619 \\
1 & 5.75 \\
2 & 10.52307155 \\
3 & 15.06421047 \\
4 & 19.4970504 \\
5 & 23.86537389 \\
\hline & $a=2$ \\
\hline 0 & -3.230518994 \\
1 & 4.510929109 \\
2 & 9.532275968 \\
3 & 14.1972814 \\
4 & 18.70978427 \\
5 & 23.13559322 \\
\hline$a_{2,0}^{(3)}=5.250535221$ \\
\hline 0 & -27.3245988 \\
1 & -0.5108147276 \\
\hline & 5.75 \\
\hline & 10.90599171 \\
\hline & 15.71422948 \\
\hline & 20.34858964 \\
\hline & \\
\hline & \\
\hline &
\end{tabular}



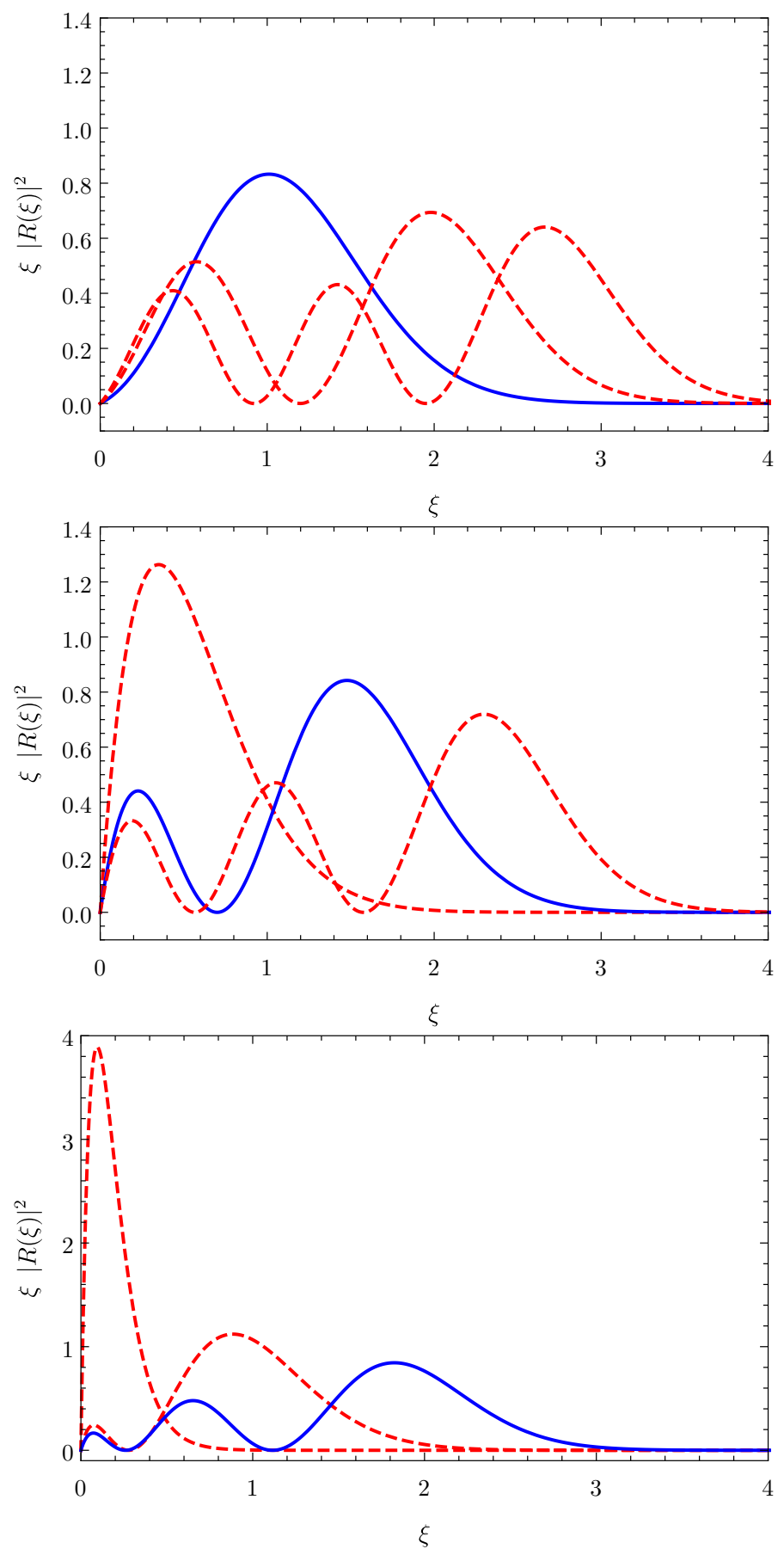

Figure 2: Functions $\xi R_{\nu, 0}(\xi)^{2}, \nu=0,1,2$, for $a_{2,0}^{(1)}=-1.940551663$ (top), $a_{2,0}^{(2)}=$ 1.190016441 (center) and $a_{2,0}^{(3)}=5.250535221$ (bottom) 


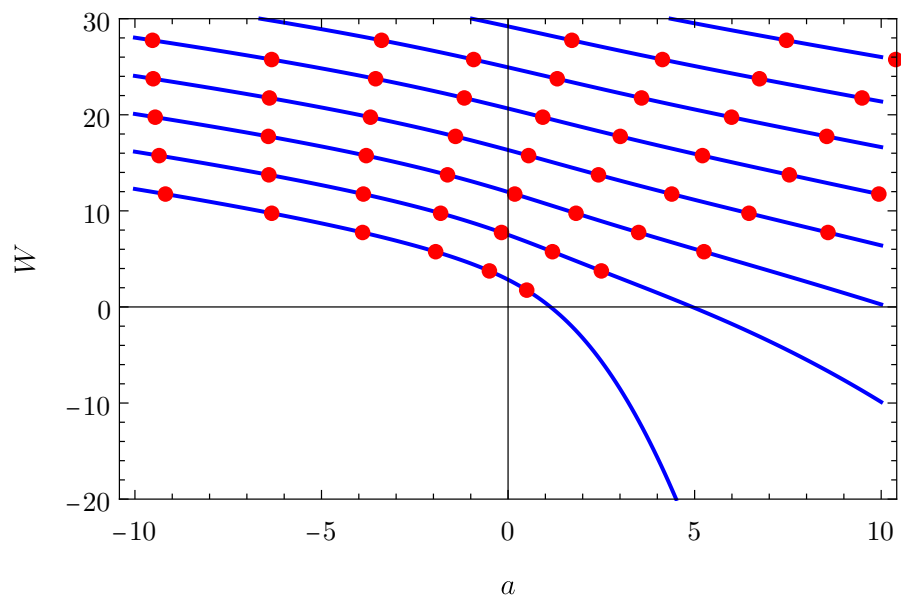

Figure 3: Eigenvalues $W_{\nu, 0}(a)$ for $b=1$ 